\title{
Hydrodynamic capture of microswimmers into sphere-bound orbits
}

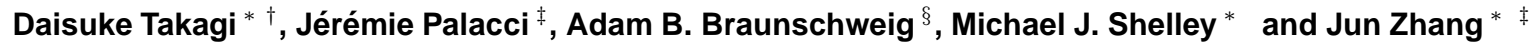 \\ *Applied Math Lab, Courant Institute, New York University, New York, New York 10012, † Department of Mathematics, University of Hawaii at Manoa, Honolulu, Hawaii \\ 96822, ${ }^{\ddagger}$ Department of Physics, New York University, New York, New York 10003, and §Department of Chemistry, University of Miami, Coral Gables, Florida 33146
}

Submitted to Proceedings of the National Academy of Sciences of the United States of America

\begin{abstract}
Self-propelled particles can exhibit surprising non-equilibrium behaviors, and how they interact with obstacles or boundaries remains an important open problem. Here we show that chemically propelled micro-rods can be captured, with little change in their speed, into close orbits around solid spheres resting on or near a horizontal plane. We show that this interaction between sphere and particle is short-range, occurring even for spheres smaller than the particle length, and for a variety of sphere materials. We consider a simple model, based on lubrication theory, of a forceand torque-free swimmer driven by a surface slip (the phoretic propulsion mechanism) and moving near a solid surface. The model demonstrates capture, or movement towards the surface, and yields speeds independent of distance. This study reveals the crucial aspects of activity-driven interactions of self-propelled particles with passive objects, and brings into question the use of colloidal tracers as probes of active matter.
\end{abstract}

active colloids | low Reynolds number | non-equilibrium physics

\section{Significance}

How autonomous microswimmers interact with their environment can be strikingly different than for passive particles, and often give rise to a wealth of non-equilibrium phenomena. We report surprising observations of synthetic swimmers being captured by, and then orbiting, spherical particles until they are kicked out by thermal fluctuations. This complex behavior can be explained in part by a simple model with the basic ingredients at work: fluid slipping on the swimmer surface and fluid coupling with nearby boundaries. Our study stresses outcomes of activity-driven interactions between swimmers and other objects that were overlooked in previous studies. Our findings reveal a richer range of possible interactions in active matter, and impact statistical physics, soft matter, fluid dynamics, and biophysics.

B iological systems demonstrate that the presence of obstacles, which could be a solid surface or a group of cells, profoundly affects the autonomous movement of motile microorganisms. Motile cells can aggregate (1) and move in circles on surfaces (2), reverse directions when they are spatially constricted (3), and migrate preferentially through an array of $\mathrm{V}$-shaped funnels (4). Bacteria may enhance the diffusivity of surrounding tracer particles (5, 6), drive ratchets into rotary motion (7, 8$)$, and form large rotating structures through collective movements (9). Interactions between bacteria and the physical environment are widely documented but complicated because, in addition to possible collisional $(10)$ and hydrodynamic (11) effects, there are unknown responses associated with behavior.

Recent technological advances have enabled the fabrication of synthetic microswimmers that convert chemical energy into directional motion $(12-16)$. Their movements can collectively drive the system out of equilibrium and exhibit large-scale phenomena such as schooling (17) and clustering (18,19). Studying the dynamics of self-propelled particles is important because they may have useful ensemble properties, inspire new designs for smart materials, and find many applications including microfluidic mixing devices and cargo transport (20-22).

Synthetic swimmers are arguably simpler than bacteria and offer insight into the effects of obstacles and confinement on self-propelled
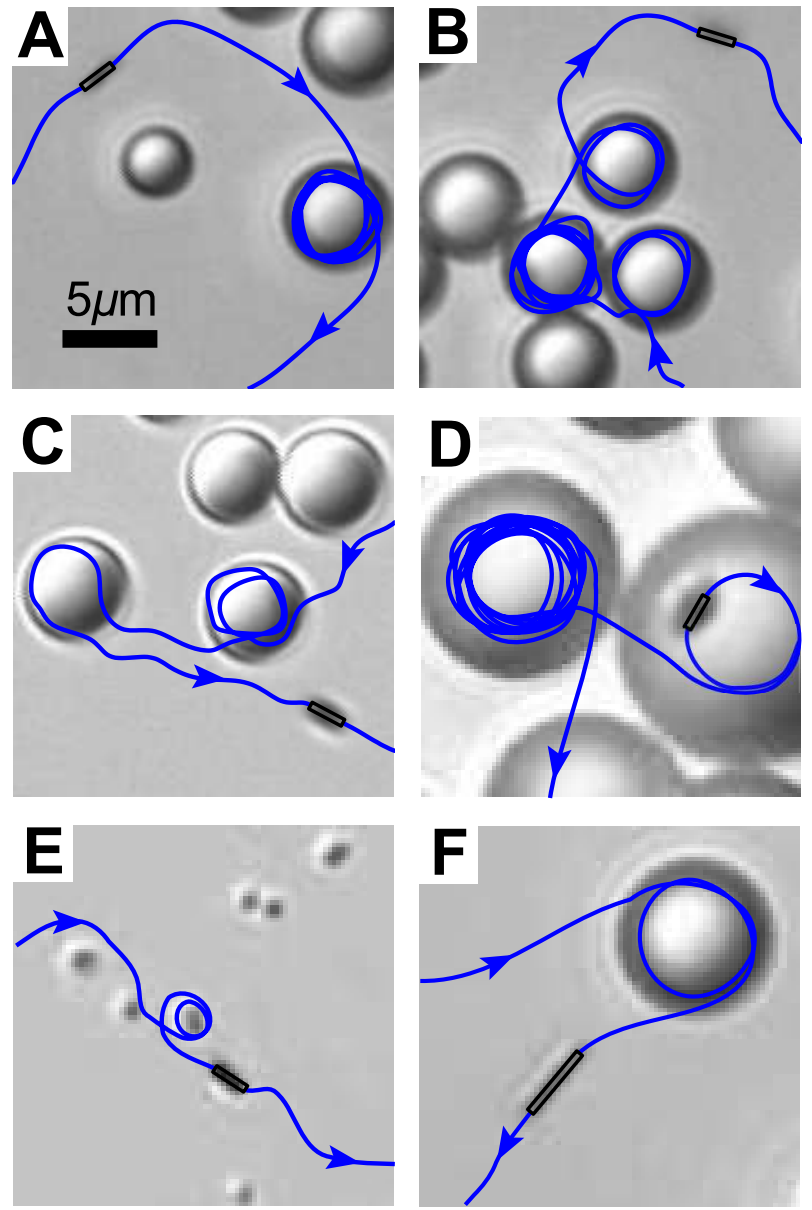

Fig. 1. Sample trajectories of self-propelled rods orbiting around passive spheres. The spheres have diameter $\sim 6 \mu \mathrm{m}$ in (A-C), 11 and $14 \mu \mathrm{m}$ in (D), $1 \mu \mathrm{m}$ in (E), and $9 \mu \mathrm{m}$ in (F), and are made of various materials (see main text). The rod length is $2 \mu \mathrm{m}$ in (A-E) and $4 \mu \mathrm{m}$ in $(F)$. The speed of the rods is on the order of $20 \mu \mathrm{m} / \mathrm{s}$.

particles. Here we employ a widely studied system consisting of goldplatinum (Au-Pt) segmented rods immersed in an aqueous hydrogen

\section{$\overline{\text { Reserved for Publication Footnotes }}$}


peroxide solution $\left(\mathrm{H}_{2} \mathrm{O}_{2}\right)$ (12). Previous studies propose that these rods propel themselves via self-electrophoresis, which generates a slip flow along the rod surface (23, 24). Recent studies show that synthetic swimmers interact with rigid boundaries, say by sliding along walls (25 26) and enhancing the diffusivity of tracer particles (27), but the mechanisms of the interactions remain unclear.

Here we show that self-propelled Au-Pt rods are captured and orbit closely, with little decrease in their speed, around solid passive spheres resting on a solid substrate (Fig. 1). We show that this interaction between rod and sphere is short range, occurs for spheres of various materials and sizes, including spheres below the rod length. While the spheres appear to attract the rods, the Stokesian fluid environment precludes any net force or torque being exerted upon them. We explain some of these observations using a simple model, based on lubrication theory, of a swimming particle moving near a wall and propelled by a surface slip. This is a suitable assumption for modeling the motion of phoretic swimmers and motile ciliates (28).

\section{Results and Discussions}

Experimental system. We fabricate Au-Pt rods with length $L=2 \pm$ $0.2 \mu \mathrm{m}$ and diameter $0.39 \pm 0.04 \mu \mathrm{m}$ following the method of electrochemical deposition in anodic aluminum oxide membranes (29) 30). They are immersed in a $\mathrm{H}_{2} \mathrm{O}_{2}$ solution with typical concentration $15 \%$ containing passive spheres, with diameters of 1 to $\sim 100 \mu \mathrm{m}$. Due to gravity, both rods and spheres remain close to, and move along, the plane of the microscope slide. The positions of the rods and spheres are tracked using optical microscopy (Nikon Eclipse 80i, $40 \times$ ), a digital camera (Lumenera Infinity 1-3), and image analysis

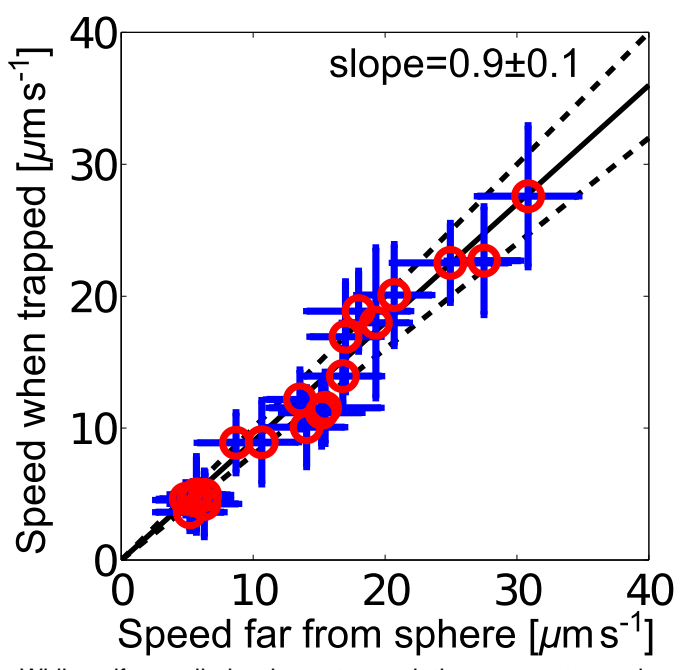

Fig. 2. While self-propelled rods are trapped, they move at speeds comparable to when they are far away from spheres. Symbols and error bars respectively show the temporal mean and standard deviation in speed of each rod. Solid and dashed lines have slopes $0.9 \pm 0.1$. Data collected at different $\mathrm{H}_{2} \mathrm{O}_{2}$ concentrations and over many events.
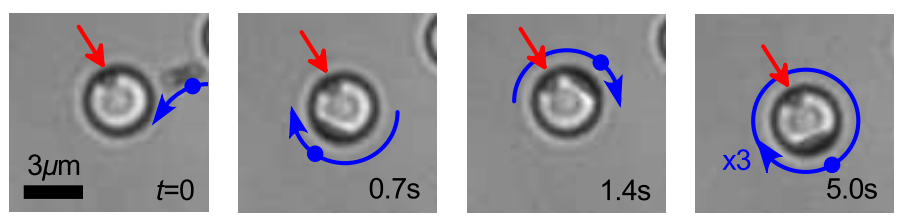

Fig. 3. Image sequence of a solid sphere of diameter $\sim 3 \mu \mathrm{m}$ which hardly rotates while a $2 \mu \mathrm{m}$-long self-propelled rod orbits around it. Red arrows point to a marker on the sphere. Blue dots and arrows show the location and direction of motion of the orbiting rod.
(Image J and Matlab). In the absence of passive spheres, the moving rods turn, flip, and disperse over time as reported previously (31).

Capture of self-propelled rods by passive spheres. The presence of passive spheres significantly alters the trajectories of self-propelled rods (Fig. 1 and Movie S1). When a self-propelled rod encounters a sphere, it typically orbits around it (Fig. 11A). The rod can move around a succession of spheres and either continue to turn in the same sense (Fig. 1 B ) or switch the handedness of its circular orbit (Fig. 11C).

This phenomenon of rods orbiting around spheres occurs across a wide range of materials and sizes (Fig. 1D-F). We have used rods of different lengths $(1,2,4 \mu \mathrm{m})$ and spheres of various diameters $(1,3,6,9,11,14,20,125 \mu \mathrm{m})$ made of glass, polymerized 3-(trimethoxysilyl)propyl methacrylate (TPM) (32), poly(methyl methacrylate) (PMMA), and polystyrene. These materials have different surface properties but this does not affect the capture effect. Self-propelled rods are captured by all spheres that remain close to the substrate by gravity, including glass beads as small as $1 \mu \mathrm{m}$ in diameter (half the rod length in Fig. 11E; see Movie S2). On a sheet of mica with cleavages which act as nearly vertical walls on a horizontal surface, the rods are transitorily trapped along the walls in a similar fashion to what has been reported before (25). This demonstrates that the rods are captured by confining walls with a variety of shapes and materials, and suggests that the capture effect may be hydrodynamic in origin.

Capture phenomenon with no additional drag. Self-propelled rods nearly maintain their typical speed while they orbit around spheres (Fig. 24). While a rod is captured, it remains in a narrow fluid region between the sphere and the horizontal glass substrate, and shows little change in speed in this confined space.

Do spheres also rotate while a rod orbits around them? To answer this we use inert TPM spheres with an embedded piece of hematite acting as a marker (33). The rotational diffusion of the sphere is slow compared to the orbiting time of the rod, and the orientation of the marker remains apparently unchanged (Fig. 3 and Movie S3). A Fourier frequency analysis of sphere displacements shows that while the sphere does fluctuate slightly because of Brownian motion, there
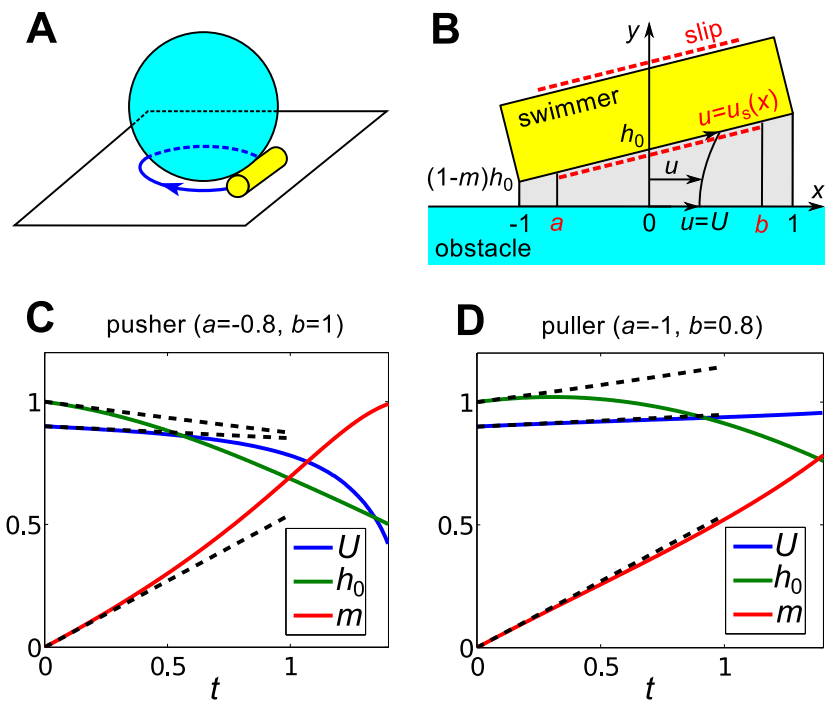

Fig. 4. (A) Sketch of a rod orbiting around a sphere on a horizontal surface. (B) Theoretical configuration of a swimming body near a rigid wall in the reference frame of the swimmer. The wall approximates the local surface of the sphere and translates to the right. Dashed lines show the region of fluid slip. (C,D) Predictions for the swimming speed $U$, distance $h_{0}$ away from the wall, and re-scaled slope $m$ of a swimmer with fluid slipping over (C) $-0.8<x<1$ and (D) $-1<x<0.8$. Dashed lines show the early-time regime (Eq. [5]7. 
is also an oscillation of the sphere at the same frequency as the rod orbital motion. However, this effect is quite small.

The nearly constant rod speed and the lack of sphere rotation suggest that the rods hardly experience any additional drag despite moving close to the solid spheres. This direct experimental evidence supports the hypothesis that the rods generate a local propulsive flow along their surface $(23,24)$ as examined in our theory below.

Tentative scenarios of capture. There are several possible mechanisms of trapping. Chemical gradients induced by fuel-consuming rods may affect rod movement. However, in our system diffusion quickly restores any local changes in fuel level because the Peclet number $P e=U L / D \sim 0.1$ is small, where $U \sim 20 \mu \mathrm{m} / \mathrm{s}$ is the typical speed, $L \sim 6 \mu \mathrm{m}$ the sphere diameter, and $D \sim 10^{3} \mu \mathrm{m}^{2} / \mathrm{s}$ the fuel diffusivity. The consumption rate of the fuel is negligible over the typical duration of our experiments. This is consistent with the swimmers having little loss in speed when orbiting a sphere. Long-range hydrodynamic effects may enable swimmers to approach a solid surface if they are represented by an extensile force dipole (pusher) (1,34), though our rods experience only short-range interactions with spheres as confirmed below. The rods are slightly curved and tend to move in curved paths with typical curvature $\kappa \sim 0.12 \mu \mathrm{m}^{-1}$ (31), so they could slide along flat (35) and curved surfaces with curvature much less than $\kappa(36)$. However, we observe rods orbiting around spheres with diameter as small as $1 \mu \mathrm{m}$, which has curvature much greater than $\kappa$.

In our interpretation, aside from thermal fluctuations, the rods move towards a surface because the local flow field is modified by its presence. This is demonstrated in a simplified model based on lubrication theory. Such a motion would not occur for an object towed by a force parallel to a solid boundary due to the symmetry and time-reversibility of Stokes flow. The key ingredient in our model is a prescribed slip on the swimming body, which captures a phoretic propulsion mechanism.

\section{Short-range hydrodynamic capture}

Mathematical model. While our experimental system is three dimensional with curved boundaries (Fig. 4A), for simplicity we consider a two-dimensional swimmer moving above a flat immobile wall (Fig. 4B). Thermal fluctuations are neglected. We adopt a Cartesian coordinate system $(x, y)$ in the reference frame that translates with the swimmer along the wall, where $x$ is scaled by a half of the length of the swimming body $L / 2$ and $y$ is scaled by a typical distance $H$ between the swimmer and the wall. The wall is represented by $y=0$ and translates to the right with speed $U$, where all speeds are scaled by a typical slip velocity $V$ on the swimmer. The wall-facing surface of the swimmer is represented by $y=h$ with $h=h_{0}(1+m x)$, $-1 \leq x \leq 1$, where $h_{0}$ is a re-scaled distance from the wall to the center of the swimmer and $m$ is a re-scaled slope of the swimmer relative to the wall. Note that $m=1$ corresponds to the front end of the swimmer (the left end in Fig. 4 4 ) hitting the wall. Our aim is to predict how $U, h_{0}$, and $m$ evolve over time, given a prescribed slip speed $u_{s}(x)$ on the surface of the swimmer.

Lubrication theory describes the fluid flow between the swimmer and the wall, provided that both the Reynolds number of the flow and $\delta=H / L$ are small. Our approach is similar to that used in modeling of crawling snails (37). The flow is primarily in the $x$ direction and governed by $d^{2} u / d y^{2}=d p / d x$, where $u$ is the flow speed, $p$ is the pressure scaled by $\mu \mathrm{VH} / L^{2}$, and $\mu$ is the dynamic viscosity of the fluid. The solution is given by

$$
u=\frac{1}{2} \frac{d p}{d x} y(y-h)+\frac{y}{h}\left(u_{s}-U\right)+U,
$$

which satisfies the boundary conditions $u=U$ on $y=0$ and $u=u_{s}(x)$ on $y=h$. Substituting Eq. 11 into the condition $\partial h / \partial t+(\partial / \partial x) \int_{0}^{h} u d y=0$ that the mass is conserved, we obtain

$$
\frac{d p}{d x}=\frac{6}{h^{3}}\left[\dot{h}_{0} x(2+m x)+\dot{m} h_{0} x^{2}+h\left(U+u_{s}\right)-2 q_{0}\right] .
$$

Here $q_{0}$ is a constant, which is obtained by integrating Eq. (2) with respect to $x$ and requiring that $p=0$ at the ends $x= \pm 1$. Eqs. (1) and (2) are used to convert the conditions that the swimmer is forceand torque-free,

$$
\left.\int_{-1}^{1} \frac{d u}{d y}\right|_{y=h} d x=\int_{-1}^{1} p d x=\int_{-1}^{1} x p d x=0,
$$

into a system of ordinary differential equations. One consequence is an expression for the swimming speed

$$
U=\frac{m}{\ln \left(\frac{1+m}{1-m}\right)} \int_{-1}^{1} \frac{u_{s}(x) d x}{1+m x},
$$

which is independent of the distance $h_{0}$ between the swimmer and the wall. In addition, no force is exerted on the wall. These are consistent with the rods nearly maintaining their speed and exerting little drag on the spheres in our experiments (Fig. 23).

We now consider a simple class of swimmers with constant slip $u_{s}=1$ on a portion of their surface $a<x<b$ and no slip $u_{s}=0$ elsewhere. This assumption is motivated by the previous hypothesis that the rods generate a local flow on their surface. At early times, a swimmer that is initially parallel to the wall with $h_{0}=1$ evolves according to the asymptotic solutions

$$
\begin{aligned}
U & \sim \frac{1}{2}(b-a)-\frac{15}{32}\left(b^{2}-a^{2}\right)\left(b-a-\left(b^{3}-a^{3}\right)\right) t, \\
h_{0} & \sim 1-\frac{3}{8}\left(b^{2}-a^{2}\right) t, \\
m & \sim \frac{15}{8}\left(b-a-\left(b^{3}-a^{3}\right)\right) t,
\end{aligned}
$$

valid to second order in time. Eq. (7) shows that, regardless of the exact region of slip, all swimmers with fluid slipping on a majority of their surface orient towards the wall. Temporal changes in $m, h_{0}$, and $U$ are shown for two typical swimmers with slip everywhere except either near its front or back end of the swimmer (Fig. 4C,D). In both cases, the swimmer approaches the wall, and its changes in speed can be related to the relative position of its no-slip region to the wall. This simple model suggests that self-propelled particles can be captured by solid surfaces without applying a mean force or torque.
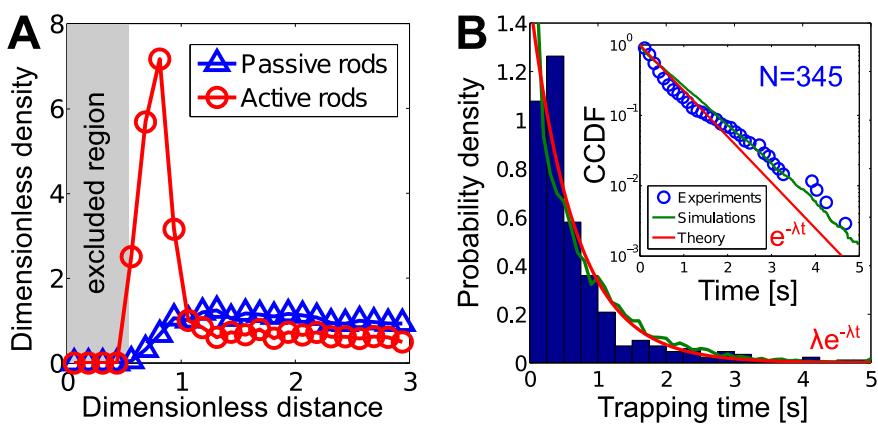

Fig. 5. (A) Experimental data of the effective density of a suspension of rods, scaled by the expectation if the rods were distributed uniformly. This is plotted against the distance from the lowest point of a sphere in units of the sphere radius, based on over 4000 measurements of the distance over time. Below the sphere, the density of passive rods falls while the density of self-propelled rods peaks. The excluded region is where the rods are physically unable to enter. (B) Normalized histogram of trapping times of self-propelled rods orbiting around spheres. Inset shows the complementary cumulative distribution function (ccdf) denoting the probability of rods remaining trapped beyond a specified time. Red curves are exponential distributions with rate parameter $\lambda=1.5 \mathrm{~s}^{-1}$. Green curves are simulated trapping times (see Materials and Methods). 
Consequences of the model. In our experiments there are effectively two walls: the spherical surface and the underlying substrate. Our theory would apply not only to the sphere as the wall but also to the substrate. Lubrication effects may induce self-propelled rods to move vertically downwards in the same direction as the gravitational force, which likely reduces the sedimentation height of self-propelled rods compared to that of diffusive rods (31). As a result the self-propelled rods orient towards the thin gap between the walls of the sphere and the substrate, though the effect may be weaker in $3 \mathrm{D}$ than in our $2 \mathrm{D}$ model.

To verify experimentally that only short-range interactions occur between self-propelled rods and passive spheres, we consider the distribution of rod positions relative to the center of a nearby sphere (Fig. 5A). This is measured by sampling the distance from a given rod to the nearest sphere over time, excluding cases where the rod has multiple spheres within three sphere radii. For passive rods in water, the density is nearly uniform everywhere except below the sphere, where the density decreases to zero. Active rods also have a uniform distribution away from the sphere, but in contrast to passive rods now show a sharp peak below the sphere. These features for self-propelled rods are consistent with the hypothesis of capture through short-range interactions. Further, hydrodynamic interactions induced by the rod's propulsive mechanism enable the spheres to capture self-propelled rods but not passive ones.

Capture time statistics. While our deterministic model predicts that swimmers move closer to a solid surface, fluctuations may cause them to move away or remain at some characteristic distance. In our experiments the trapping is transitory, and we observe that the trapping times are typically less than 5 seconds and have an exponential distribution with decay rate $\lambda \sim 1.5 \mathrm{~s}^{-1}$ (Fig. $5 \mathrm{~B}$ ). The trapping time-scale $\left(\lambda^{-1}\right)$ is comparable to the time-scales of rotational diffusion and duration between stochastic flips $(\sim 1 \mathrm{~s})(31)$.

In rare instances, the rods remain trapped on the order of minutes. Highly curved rods that move in small circles tend to either remain trapped for relatively long times or are hardly trapped, depending on whether they approach a sphere with the trajectory curving towards or away from the sphere, respectively. One possible interpretation of this is that the rods are curved towards the sphere while they are trapped until thermal fluctuations flip them over (31) and enable them to escape.

We have developed a numerical model by including thermal fluctuations in the orientational dynamics and defining a critical angle for escape (see Materials and Methods). This yields a Poisson process for escape times, and reproduces the exponential distribution found in Fig. 5B. The relative residence times of trapped $v s$ untrapped parti- cles would then yield the relative densities in Fig. 54. While thermal fluctuations interacting with the capture effect seem to explain the basic mechanisms at work, a more predictive theory should include swimmer geometry and detailed chemistry.

\section{Conclusion}

Suspensions of self-propelled and passive particles reveal new aspects of dynamics in active matter. Swimmers with an effective slip on a majority of their surface, including phoretic particles (23, 24) and motile ciliates (28), move without causing much disturbance to colloidal particles. This suggests that phoretic particles can move stealthily, with little perturbation to the surrounding fluid and objects within it. Still, the swimmers themselves are captured by solid boundaries and can accumulate in spatially constricted regions, unlike passive particles. This may offer insight into the hydrodynamic effects in cell adhesion and feeding, as well as suggest strategies to filter groups of swimmers through the confined spaces of capillaries, microfluidic devices, and porous media. Our results also bring into question the use of colloidal tracers as probes in out-of-equilibrium systems, as for example in measuring the effective temperature of an active bath (5, 6, 27). The tracers may not fully capture the complex swimmer movements and enhanced fluid mixing which could emerge in concentrated suspensions. We speculate that adding a payload to phoretic swimmers such as studied here would markedly affect how they interact with the surrounding fluid and with obstacles.

ACKNOWLEDGMENTS. We thank S. Sacanna for the hematite-encapsulating spheres used in this study. We acknowledge support from NSF (NYU MRSEC DMR-0820341, MRI-0821520, DMR-0923251, DMS-0920930), DOE (DE-FG0288ER25053), and AFOSR (FA-9550-11-1-0032).

\section{Materials and Methods}

Simulation of trapping times. To study the effect of thermal fluctuations on the trapping time, we carried out simple simulations of our model with the added effect of rotational Brownian motion. Consider a swimmer with fluid slip over $90 \%$ of its body length $L=2 \mu \mathrm{m}$ ( $a=-0.9, b=0.9$ ) moving at speed $U=20 \mu \mathrm{m} / \mathrm{s}$ at a typical distance $\mathrm{H} \sim 0.2 \mu \mathrm{m}$ from the wall. We simulate the orientation angle $\theta$ of 10000 swimmers which are initially parallel to a wall. The angle evolves according to the Langevin Equation

$$
\theta(t+\Delta t)=\theta(t)+\Omega \Delta t+\sqrt{2 D_{r} \Delta t} X,
$$

where $\Omega \sim 3 / \mathrm{s}$ is the typical rotation rate estimated using Eq. 7$] D_{r}=1 / \mathrm{s}$ is the rotational diffusion constant, and $X$ is a random variable with normal distribution. This shows that the trapping time, defined as the time it takes for the angle to decrease to a threshold value $-\pi / 9$, is exponentially distributed in agreement with our experimental data (Fig. [53).
1. Berke AP, Turner L, Berg HC, Lauga E (2008) Hydrodynamic attraction of swimming microorganisms by surfaces. Phys Rev Lett 101:38102.

2. Lauga E, DiLuzio WR, Whitesides GM, Stone HA (2006) Swimming in circles: motion of bacteria near solid boundaries. Biophys $J$ 90:400-412.

3. Cisneros L, Dombrowski C, Goldstein RE, Kessler JO (2006) Reversal of bacterial locomotion at an obstacle. Phys Rev E 73:030901.

4. Galajda P, Keymer J, Chaikin P, Austin R (2007) A wall of funnels concentrates swimming bacteria. J Bacteriol 189:8704-8707.

5. Wu XL, Libchaber A (2000) Particle diffusion in a quasi-two-dimensional bacterial bath. Phys Rev Lett 84:3017-3020.

6. Leptos KC, Guasto JS, Gollub JP, Pesci Al, Goldstein RE (2009) Dynamics of enhanced tracer diffusion in suspensions of swimming eukaryotic microorganisms. Phys Rev Lett 103:198103.

7. Di Leonardo R, et al. (2010) Bacterial ratchet motors. Proc Natl Acad Sci 107:95419545.

8. Sokolov A, Apodaca MM, Grzybowski BA, Aranson IS (2010) Swimming bacteria power microscopic gears. Proc Natl Acad Sci 107:969-974.

9. Schwarz-Linek J, et al. (2012) Phase separation and rotor self-assembly in active particle suspensions. Proc. Natl Acad Sci 109:4052-4057.

10. Kantsler V, Dunkel J, Polin M, Goldstein RE (2013) Ciliary contact interactions dominate surface scattering of swimming eukaryotes. Proc Natl Acad Sci 110:1187-1192.
11. Drescher K, Dunkel J, Cisneros LH, Ganguly S, Goldstein RE (2011) Fluid dynamics and noise in bacterial cell-cell and cell-surface scattering. Proc Natl Acad Sci 108:10940-10945.

12. Paxton WF, et al. (2004) Catalytic nanomotors: Autonomous movement of striped nanorods. J Am Chem Soc 126:13424-13431.

13. Fournier-Bidoz S, Arsenault AC, Manners I, Ozin GA (2005) Synthetic self-propelled nanorotors. Chem Commun pp 441-443.

14. Gibbs JG, Zhao YP (2009) Design and characterization of rotational multicomponent catalytic nanomotors. Small 5:2304-2308.

15. Ebbens SJ, Howse JR (2010) In pursuit of propulsion at the nanoscale. Soft Matter 6:726-738.

16. Sengupta S, Ibele ME, Sen A (2012) Fantastic voyage: Designing self-powered nanorobots. Angew Chem Int Ed 51:8434-8445.

17. Ibele M, Mallouk TE, Sen A (2009) Schooling behavior of light-powered autonomous micromotors in water. Angew Chem Int Ed 48:3308-3312.

18. Theurkauff I, Cottin-Bizonne C, Palacci J, Ybert C, Bocquet L (2012) Dynamic clustering in active colloidal suspensions with chemical signaling. Phys Rev Lett 108:268303.

19. Palacci J, Sacanna S, Steinberg AP, Pine DJ, Chaikin PM (2013) Living crystals of light-activated colloidal surfers. Science 339:936-940.

20. Pumera M (2011) Nanomaterials meet microfluidics. Chem Commun 47:5671-5680. 
21. Wang J, Gao W (2012) Nano/microscale motors: Biomedical opportunities and challenges. ACS Nano 6:5745-5751.

22. Patra $D$, et al. (2013) Intelligent, self-powered, drug delivery systems. Nanoscale.

23. Wang $Y$, et al. (2006) Bipolar electrochemical mechanism for the propulsion of catalytic nanomotors in hydrogen peroxide solutions. Langmuir 22:10451-10456.

24. Moran JL, Posner JD (2011) Electrokinetic locomotion due to reaction-induced charge auto-electrophoresis. J Fluid Mech 680:31-66.

25. Volpe G, Buttinoni I, Vogt D, Kümmerer HJ, Bechinger C (2011) Microswimmers in patterned environments. Soft Matter 7:8810-8815.

26. Thutupalli $S$ (2011) Towards autonomous soft matter systems: experiments on membranes and active emulsions. PhD thesis (University of Göttingen, Germany).

27. Mino G, et al. (2011) Enhanced diffusion due to active swimmers at a solid surface. Phys Rev Lett 106:48102.

28. Stone HA, Samuel ADT (1996) Propulsion of microorganisms by surface distortions. Phys Rev Lett 77:4102-4104.

29. Martin BR, et al. (1999) Orthogonal self-assembly on colloidal gold-platinum nanorods. Adv Mater 11:1021-1025.

30. Banholzer MJ, Qin L, Millstone JE, Osberg KD, Mirkin CA (2009) On-wire lithography: synthesis, encoding and biological applications. Nat Protocols 4:838-848.
31. Takagi D, Braunschweig AB, Zhang J, Shelley MJ (2013) Dispersion of self-propelled rods undergoing fluctuation-driven flips. Phys Rev Lett 110:038301.

32. Sacanna S, Irvine WTM, Rossi L, Pine DJ (2010) Lock and key colloids through polymerization-induced buckling of monodisperse silicon oil droplets. Soft Matter 7:1631-1634.

33. Sacanna S, Rossi L, Pine DJ (2012) Magnetic click colloidal assembly. J Am Chem Soc 134:6112.

34. Spagnolie S, Lauga E (2012) Hydrodynamics of self-propulsion near a boundary: predictions and accuracy of far-field approximations. J Fluid Mech 700:105-147.

35. van Teeffelen S, Löwen H (2008) Dynamics of a Brownian circle swimmer. Phys Rev E 78:020101.

36. van Teeffelen S, Zimmermann U, Löwen H (2009) Clockwise-directional circle swimmer moves counter-clockwise in petri dish-and ring-like confinements. Soft Matter 5:4510-4519.

37. Chan B, Balmforth NJ, Hosoi AE (2005) Building a better snail: Lubrication and adhesive locomotion. Phys Fluids 17:113101. 